Trinity College

Trinity College Digital Repository

Faculty Scholarship

7-2016

Search for anomalies in the decay of radioactive Mn-54

Mark P. Silverman

Trinity College, mark.silverman@trincoll.edu

Follow this and additional works at: https://digitalrepository.trincoll.edu/facpub

Part of the Physical Sciences and Mathematics Commons 


\title{
Search for anomalies in the decay of radioactive $\mathrm{Mn}-54$
}

\author{
M. P. Silverman ${ }^{(a)}$ \\ Department of Physics, Trinity College - Hartford, CT 06106, USA
}

received 29 March 2016; accepted in final form 20 June 2016

published online 12 July 2016

PACS 23.40.-s - $\beta$ decay; double $\beta$ decay; electron and muon capture

PACS 24.60.-k - Statistical theory and fluctuations

PACS 29.85.Fj - Data analysis

\begin{abstract}
Recent papers have reported that ${ }^{54} \mathrm{Mn}$, which decays by electron capture (a weak nuclear interaction) with half-life $\sim 312$ days, is influenced by solar activity. Should this actually occur, new physics would be needed to explain it. This paper reports results of an analysis of ${ }^{54} \mathrm{Mn}$ activity measured over a time interval of $\sim 3.6$ half-lives. If standard nuclear physics applies, the logarithmic residuals of ${ }^{54} \mathrm{Mn}$ activities should form a stationary set of independent random variables whose statistics are determined solely by a constant decay rate $\lambda$ and initial mean count $\mu$. Analysis of the time-variation, autocorrelation, and power spectra of the ${ }^{54} \mathrm{Mn}$ logarithmic residuals agrees exquisitely with standard nuclear physics. Computer-simulated activities exhibiting periodic decay of amplitude $A=\alpha \lambda$ show that anomalies would be detectable by these statistical tests for values of $\alpha$ as low as $\sim 1$ part in $10^{4}$. This limit is about 10 times lower than reported deviations from exponential decay.
\end{abstract}

Introduction. - In recent years there have been reports of anomalous radioactive processes such as nonexponential decay, periodic decay rates, correlation of fluctuations in decay rates, and other observations at variance with the laws of physics as they are currently understood. (For references and discussion, see [1].) These reports are controversial; there is no consensus among physicists regarding the existence of such phenomena. For example, a comprehensive search for correlated fluctuations in the decay of $\beta^{+}$emitter ${ }^{22} \mathrm{Na}$ led to no evidence for such correlations, but to excellent agreement with standard nuclear physics [2]. Likewise, a study of the power output of the Cassini spacecraft radioisotope thermoelectric generators led to no significant deviations in the exponential decay law [3].

${ }^{54} \mathrm{Mn}$, which transmutes to ${ }^{54} \mathrm{Cr}$ (branching ratio $\sim 99.997(3) \%$ ) by orbital electron capture with a half-life of about 312 days [4], is among the nuclei reported to show effects due to solar activity [5-8]. This is particularly interesting because capture of K-shell or L-shell electrons is susceptible to environmental influences that modify the electron density at the nucleus [9]. Nevertheless, it would be difficult, if not impossible, to understand the reported effect of the Sun on an Earth-bound ${ }^{54} \mathrm{Mn}$ nucleus according to the theory of electro-weak

\footnotetext{
(a) E-mail: mark.silverman@trincoll.edu
}

interactions and its specific application to electron capture theory $[10]$.

The controversy over environmental effects on nuclear decay is of fundamental importance. If such phenomena occur and are not instrumental artifacts, they would likely require revision of known physical laws or introduction of new ones. Electron capture is especially significant because it contributes to the decay of some 500 radionuclides and is relevant to fundamental issues of nuclear physics, astrophysics, cosmology, and geochemistry [9].

This paper reports a comprehensive statistical analysis of the activity of ${ }^{54} \mathrm{Mn}$ measured in a Centronic IG12/A20 ionisation chamber over a period of about 3.6 half-lives from 2006 to 2009. Data were provided by the Institute for Reference Materials and Measurements (IRMM) of the European Commission Joint Research Centre. Details of the experiment, originally undertaken to reduce uncertainties in the ${ }^{54} \mathrm{Mn}$ half-life, were reported in [11]. In the analysis reported here, the non-stationary time series of ${ }^{54} \mathrm{Mn}$ activities (currents measured in $\mathrm{pA}$ ) was transformed into a stationary set of logarithmic residuals -i.e., time-independent differences between observed and theoretically expected values of the log of activity. As shown previously in [12], time series of nuclear activities comprise a mixed Poisson-Gauss (MPG) distribution of random variables if the hypothesis that radioactive nuclei decay at a constant rate uninfluenced by environmental 


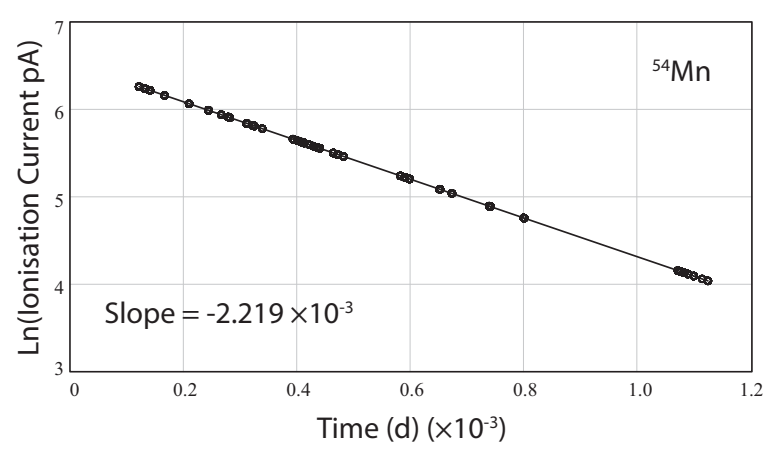

Fig. 1: Log of ionisation current (in pA) (open circles) superposed by maximum likelihood (ML) fit (solid line). Data comprise $N=19370$ points measured discontinuously over $\sim 3 \mathrm{y}$. ML slope is $\left(2.219 \pm 1.8 \times 10^{-5}\right) \times 10^{-3} \mathrm{~d}^{-1}$ corresponding to a half-life of $\sim 312$ days.
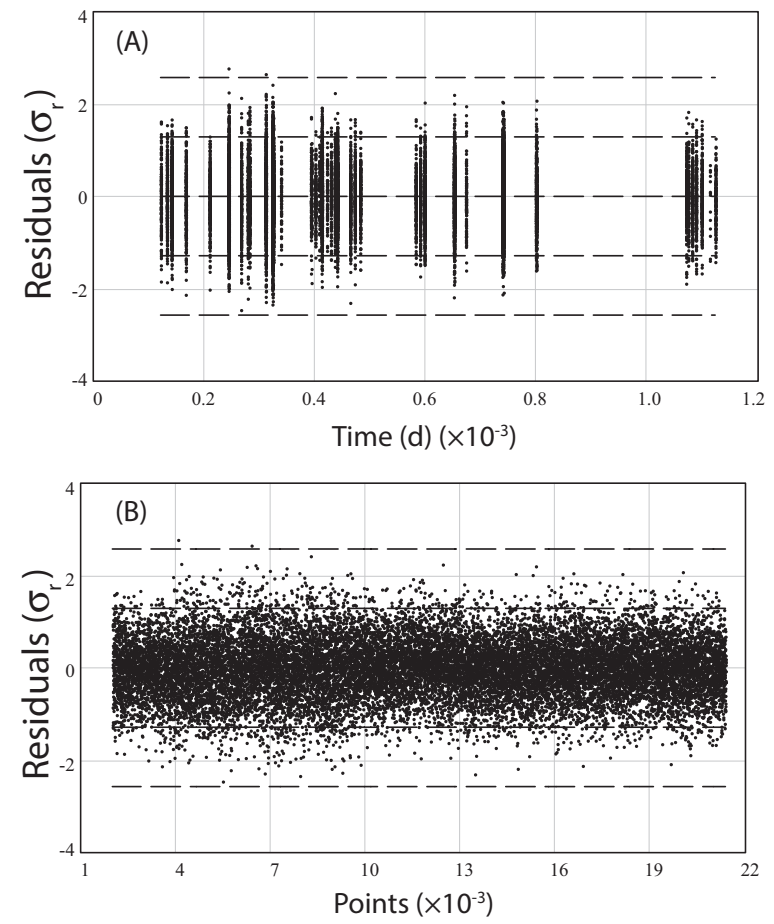

Fig. 2: Residuals (in units of $\sigma_{r}$ ) as a function of (A) time (d) and (B) point number. Dashed lines mark ordinate 0 and confidence intervals $\pm 2 \sigma_{r}, \pm 4 \sigma_{r}$ where $\sigma_{r}=6.432 \times 10^{-4}$ is the ML estimate.

interactions is valid. Residuals and certain functions of the residuals examined in this paper are particularly sensitive to violations of this hypothesis of standard nuclear decay.

Time series of ${ }^{54} \mathrm{Mn}$ activities. - Details of ${ }^{54} \mathrm{Mn}$ activity measurement by means of an ionisation chamber are given in [11]. For the analysis reported here, the time series $\left\{x_{n} n=0 \ldots N-1\right\}$ of ${ }^{54} \mathrm{Mn}$ decays comprised $N=19370$ measurements ("points") accumulated discontinuously over a period of $T=1134$ days. Figure 1 shows the series $\left\{\ln x_{n}\right\}$ as a function of measurement

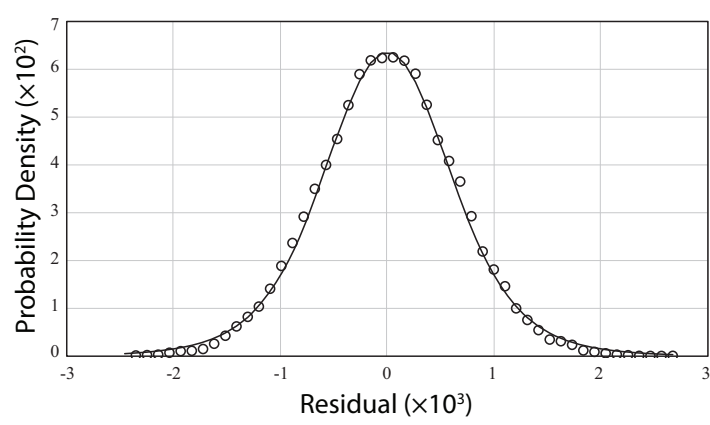

Fig. 3: Distribution of residuals of $\ln x$ (open circles) where activity $x$ is measured as an ionisation current. The solid trace is the theoretical pdf (3). The histogram comprises 19370 events partitioned in 50 bins of width $1.047 \times 10^{-4}$.

times $\left\{t_{n}\right\}$; open circles mark when measurements were made; the solid line is the maximum-likelihood (ML) line of regression $y_{n}=-\lambda t_{n}+y_{*}$ yielding a slope of magnitude $\lambda=\left(2.219 \pm 1.8 \times 10^{-5}\right) \times 10^{-3} \mathrm{~d}^{-1}$ [halflife $\left.\tau_{1 / 2}=\ln 2 / \lambda=\left(312.37 \pm 2.6 \times 10^{-3}\right) \mathrm{d}\right]$, and intercept $y_{*}=\left(6.529 \pm 1.0 \times 10^{-5}\right)$. (Reference [11] reports a slightly different half-life based on a different analytic method.) Residuals are defined in this paper by

$$
d_{n}=\ln x_{n}-y_{n} \quad(n=0 \ldots N-1) .
$$

Figure 2 shows residuals plotted as a function of (A) measurement time $t_{n}$ and (B) point number $n$. (An effective conversion of 26.59 points per day is established from fig. 11 of the final section which displays a simulated periodic 360 day autocorrelation in points.) If the hypothesis of standard nuclear decay is valid, $\left\{d_{n}\right\}$ should comprise to good approximation a stationary set of Gaussian random variables of variance

$$
\sigma_{d}^{2}=N^{-1} \sum_{n}\left(\ln x_{n}-y_{n}\right)^{2}=\left(6.432 \times 10^{-4}\right)^{2} .
$$

Horizontal dashed lines mark theoretical confidence limits $\pm 2 \sigma_{d}$ and $\pm 4 \sigma_{d}$

The distribution of residuals is shown in fig. 3, superposed (solid line) by the exact theoretical probability density function (pdf) derived from the theory of MPG distributions developed in [1] and [12]

$$
P_{\text {res }}(z)=N^{-1} \sum_{n}\left(\frac{\mu e^{-\lambda t_{n}}}{2 \pi}\right)^{\frac{1}{2}} \exp \left(-\frac{1}{2} \mu z^{2} e^{-\lambda t_{n}}\right) \text {. }
$$

Continuous variable $z$ quantifies the residuals; $\mu$ is the effective mean initial decay count (i.e. count rate $\times$ sampling interval). With $\lambda$ estimated from the decay curve, a fit of pdf (3) to the distribution in fig. 3 yielded the parameter $\mu=(6.96 \pm 0.05) \times 10^{6}$. Agreement between theory and observation in fig. 3 is excellent; a chi-square test yielded $p_{\text {res }}\left(\chi_{28}^{2}\right)=0.535$. A plot of the normal distribution $N\left(0, \sigma_{d}^{2}\right)$ closely overlaps the exact pdf $(3)$ and is not shown in the figure. Note: A $p$-value is the probability 

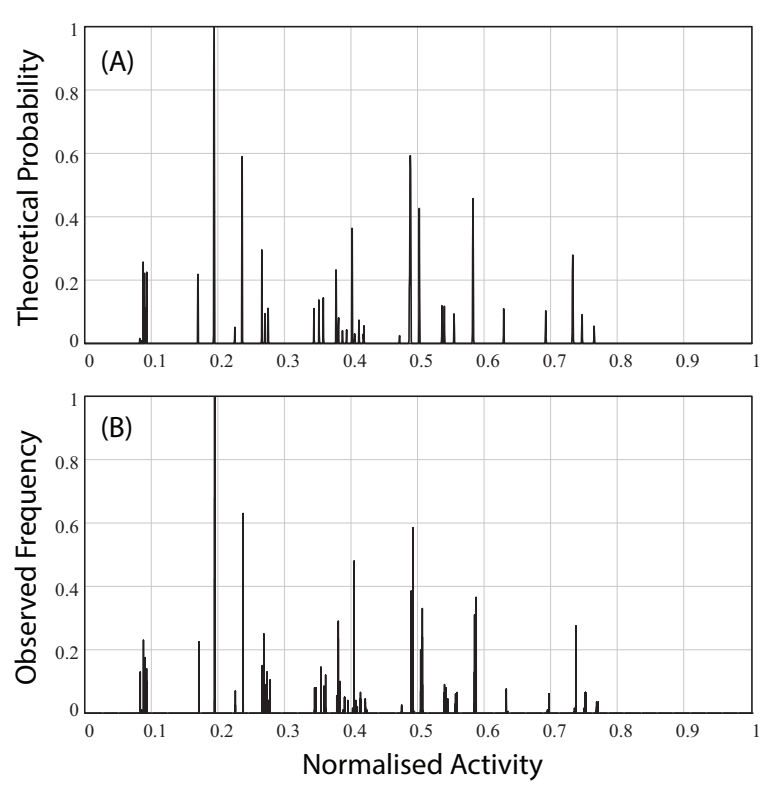

Fig. 4: Comparison of theoretical and observed distributions of activity. (A) MPG pdf (4) with ML parameters $\lambda=$ $0.00222 \mathrm{~d}^{-1}$ and $\mu=6.96 \times 10^{6}$. (B) Histogram of 19370 activities distributed over 2000 bins.

that in subsequent trials $\chi_{q}^{2} \geq$ the observed value $\left(\chi_{q}^{2}\right)_{\text {obs }}$; $q$ is the number of degrees of freedom. (For fig. $3, q=30$ bins of sufficient events -2 constraints.) For details on use and interpretation of $p$-values, see [13].

Given $\mu$ and $\lambda$, one can predict the distribution of activities from the MPG probability function derived in $[1,12]$.

$$
\begin{aligned}
P_{\mathrm{MPG}}(s)= & N^{-1} \sum_{n}\left(2 \pi \mu e^{-\lambda t_{n}}\right)^{-\frac{1}{2}} \\
& \times \exp \left(-\mu\left(s-e^{-\lambda t_{n}}\right)^{2} / 2 e^{-\lambda t_{n}}\right) .
\end{aligned}
$$

The continuous variable $s=x / \mu$ spans the range $(1 \geq s \geq 0)$. In the case of a continuous, uniform time series of activities with $\mu \gg 1$, a plot of $P_{\mathrm{MPG}}(s)$ yields a smooth Gaussian-like curve for times $\lambda T \ll 1$ that evolves into a curve $\propto s^{-1}$ in the limit $\lambda T \gg 1$, as shown in [12] and [1]. Figures $4(\mathrm{~A})$ and $(\mathrm{B})$, respectively, show the theoretical and observed distributions in the present case of a discontinuous, non-uniform time series of ${ }^{54} \mathrm{Mn}$ activities. The location and relative amplitude of lines are in excellent accord.

Squares of residuals. - In fig. 5(A) the squares of residuals (1) normalised by twice the variance,

$$
D_{n}=d_{n}^{2} / 2 \sigma_{d}^{2}
$$

are plotted as a function of time. From statistical transformations derived in [13], it can be shown that, if $d$ is a normal random variable $N\left(0, \sigma_{d}^{2}\right)$, then to good approximation $D$ is a gamma random variable $G(a, b)=G\left(1, \frac{1}{2}\right)$
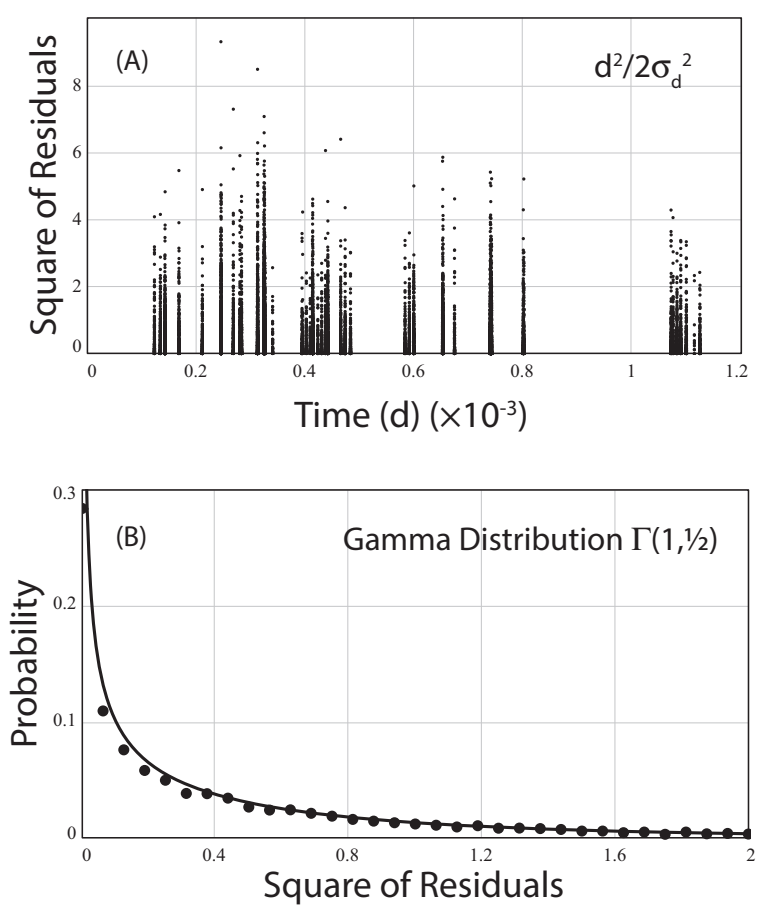

Fig. 5: (A) Normalised square of residuals $D=\left\{d^{2} / 2 \operatorname{var}\left(d^{2}\right)\right\}$. (B) Histogram of $D$ (black dots) superposed by $G\left(1, \frac{1}{2}\right)$ pdf $(6)$ (solid line).

with pdf

$p_{\mathrm{G}}(w \mid a, b)=\Gamma(b)^{-1} a^{b} w^{b-1} e^{-a w} \underset{\substack{a \\ b \\ b=1 / 2}}{\stackrel{1}{\longrightarrow}} \pi^{-\frac{1}{2}} w^{-\frac{1}{2}} e^{-w}$.

In fig. $5(\mathrm{~B})$ is plotted a histogram of $\left\{D_{n}\right\}$ superposed by theoretical pdf (6). Measurement and theory are in excellent agreement.

Autocorrelation of residuals. - The sample autocovariance of lag $k$ can be defined in various ways [14], but is here taken in its simplest form

$$
c_{k}=N^{-1} \sum_{n=0}^{N-k-1}\left(d_{n} d_{n+k}\right) \quad(k=0 \ldots K)
$$

for a time series of zero mean and zero trend. The maximum lag $K$ (in points) is somewhat arbitrary, but must be significantly less than $N$ if eq. (7) is to be useful. For larger $K$, the factor $N^{-1}$ in (7) is to be replaced by $(N-k)^{-1}$. In this section $K=10000$; other choices do not change the statistical results. If the hypothesis of standard nuclear decay is valid, the sample autocorrelation $r_{k}=c_{k} / c_{0}\left(1 \geq r_{k} \geq-1\right)$ is to good approximation a Gaussian random variable, $r_{k}=$ $N\left(\mu_{r}, \sigma_{r}^{2}\right)=N\left(-N^{-1}, N^{-1}\right)[13,14]$. For $N \gg 1$, the mean $\mu_{r} \approx 0$ for all practical purposes.

Figure 6 shows a plot of $\left\{r_{k}\right\}$, with lag $k$ in units of point number; dashed lines mark confidence limits $\pm 2 \sigma_{r}$. The number of sample autocorrelations falling 


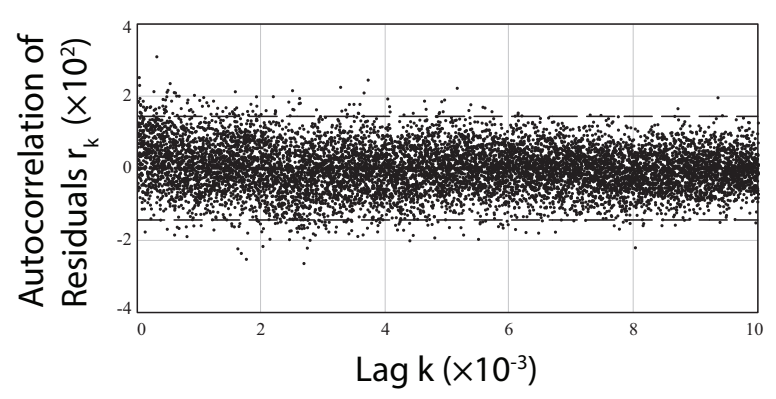

Fig. 6: Autocorrelation $\left\{r_{k}=c_{k} / c_{0}\right\}$ of residuals as a function of lag $k$ (in units of point number). Dashed lines mark ordinates $0, \pm 2 \sigma_{r}$ with $\sigma_{r} \approx N^{-\frac{1}{2}}$.

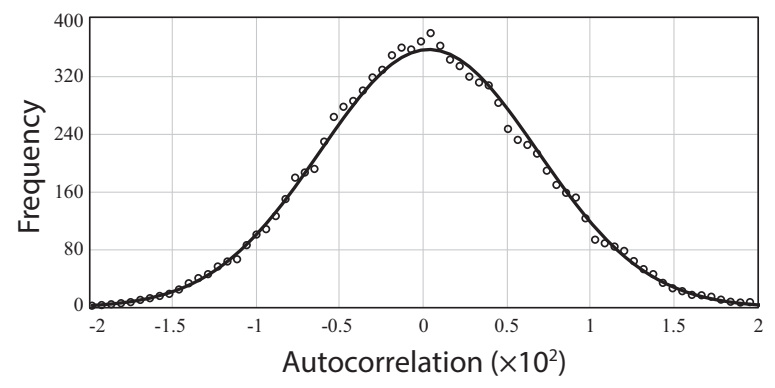

Fig. 7: Distribution of autocorrelation of residuals (open circles) superposed by Gaussian pdf $N\left(\mu_{r}, \sigma_{r}^{2}\right)$ (solid line) with $\mu_{r} \pm \sigma_{r}=(3.0 \pm 6.5) \times 10^{-3}$. The histogram comprises 19370 events distributed over 100 bins of width $5.79 \times 10^{-4}$.

outside the $99.7 \%$ confidence band $\left(-3 \sigma_{r},+3 \sigma_{r}\right)$ is 15 , which does not exceed the number due to pure chance $(1-\operatorname{erf}(3 / \sqrt{2})) K=27$ expected if the observations are independently distributed in accord with standard nuclear decay.

A histogram of $\left\{r_{k}\right\}$ (open circles) is shown in fig. 7, superposed by the theoretical Gaussian density function (solid line), centred on 0 with width $\sigma_{r}=6.5 \times 10^{-3}$ in reasonable accord with the approximate theoretical value $N^{-\frac{1}{2}}=7.2 \times 10^{-3}$. A chi-square test of the fit yielded $p\left(\chi_{68}^{2}\right)=0.999$.

Power spectrum of residuals. - A discrete time series of length $N \Delta t$ can be characterised as a discrete function of frequency $\nu_{j}=j \nu_{\mathrm{f}}$, with fundamental $\nu_{\mathrm{f}}=$ $(N \Delta t)^{-1}$ and harmonic numbers $j=0 \ldots \frac{1}{2} N$. The Nyquist or cut-off frequency is $\nu_{\mathrm{c}}=(2 \Delta t)^{-1}$, above which aliasing occurs. Because elements of a stochastic process are random variables, it is the measurable power spectrum, rather than Fourier transform, that is of primary interest. There are various ways to estimate the power spectrum, some better suited than others, depending on the properties of the parent series. Analysts recommend that several methods be tried before drawing conclusions regarding spectral content [15].

To search for evidence of periodic forcings in the decay of ${ }^{54} \mathrm{Mn}$, the discrete power spectrum $\left\{S_{j}\right\}$ of the residuals was calculated in four ways:

1) Wiener-Khintchine (WK) relation $[16]$

$$
S_{j}^{(\mathrm{WK})}=\pi^{-1}\left[c_{0}+2 \sum_{k=1}^{K} c_{k} \cos \left(2 \pi j k N^{-1}\right)\right] .
$$

2) Wiener-Khintchine relation with a Blackman-Tukey lag window [17]

$$
\begin{aligned}
S_{j}^{(\mathrm{BT})} & =\pi^{-1}\left[w_{0} c_{0}+2 \sum_{k=1}^{K} w_{k} c_{k} \cos \left(2 \pi j k N^{-1}\right)\right] \\
w_{k} & =\frac{1}{2}\left(1+\cos \left(\pi k K^{-1}\right)\right) .
\end{aligned}
$$

3) Direct Fourier transform of residuals

$$
S_{f}^{(\mathrm{FT})}=(\pi N)^{-1}\left|\sum_{n=0}^{N-1} d_{n} \exp \left(i 2 \pi f t_{n} T^{-1}\right)\right|^{2} .
$$

4) Fast Fourier transform of residuals with CooleyTukey algorithm [18],

$$
\begin{aligned}
S_{j^{\prime}}^{(\mathrm{FFT})}= & M^{-1}\left|\sum_{m=0}^{M-1} d_{m} \exp \left(2 \pi i j^{\prime} m M^{-1}\right)\right|^{2} \\
& \left(M=2^{14} ; j^{\prime}=0 \ldots \frac{1}{2} M\right) .
\end{aligned}
$$

Some clarifying remarks about the four spectra: a) The unit of frequency in spectra $S_{j}^{(\mathrm{WK})}, S_{j}^{(\mathrm{BT})}$ is cycles per point where $N=19370$ points. Likewise for spectrum $S_{j^{\prime}}^{(\mathrm{FFT})}$, except that the FFT algorithm requires the length of the parent series to be an integer power of $2 ; M=16384$ is the largest integer of this form less than $N$. b) The unit of frequency in spectrum $S_{f}^{(\mathrm{FT})}$ is cycles per day. c) Since sets $\left\{d_{n}\right\}$ and $\left\{c_{k}\right\}$ are finite, spectra $S_{j}^{(\mathrm{WK})}, S_{j}^{(\mathrm{FT})}, S_{j^{\prime}}^{(\mathrm{FFT})}$ correspond to convolving the true power spectrum with a boxcar function (i.e., difference of two Heaviside step functions), which can distort the spectrum. Use of lag window (10) avoids the sharp cut-offs of the boxcar function; thus spectrum $S_{j}^{(\mathrm{BT})}$ incurs less power loss through side lobes of the Fourier transform of the window function.

All four methods generated spectra that were essentially equivalent physically. Figure 8 shows spectrum $S_{j^{\prime}}^{(\mathrm{FFT})}$ as an example. The frothy appearance is characteristic of white noise in which the sample standard deviation of the spectrum is comparable to the mean [2]. For fig. 8 one finds $\left\langle S^{(\mathrm{FFT})}\right\rangle=3.755 \times 10^{-7}$ and $\left\langle\left(S^{(\mathrm{FFT})}-\left\langle S^{(\mathrm{FFT})}\right\rangle\right)^{2}\right\rangle^{\frac{1}{2}}=3.743 \times 10^{-7}$. If residuals $\left\{d_{n}\right\}$ are $N\left(0, \sigma_{d}^{2}\right)$ Gaussian random variables, then the power spectrum of the residuals should comprise samples of an exponential distribution $S=E\left(\sigma_{d}^{2}\right)$ with pdf

$$
P_{\exp }\left(s \mid \mu_{s}\right)=\mu_{s}^{-1} e^{-s / \mu_{s}},
$$




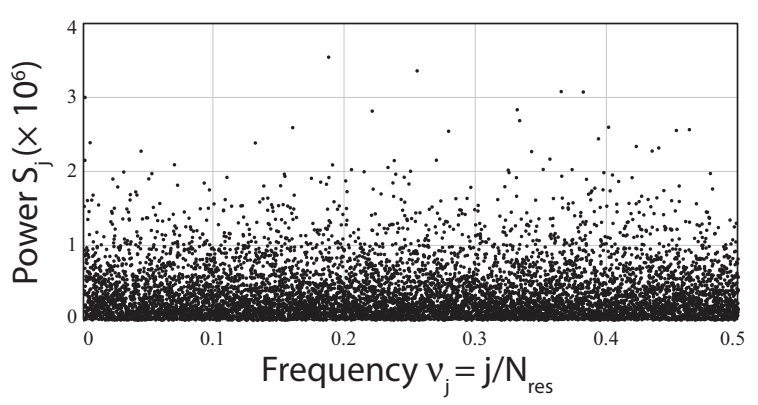

Fig. 8: Power spectrum $S_{j}^{(\mathrm{FFT})}$ of residuals computed by FFT. Sample size of residuals is $M=2^{14}$; number of FFT power spectral amplitudes is $\frac{1}{2} M$.

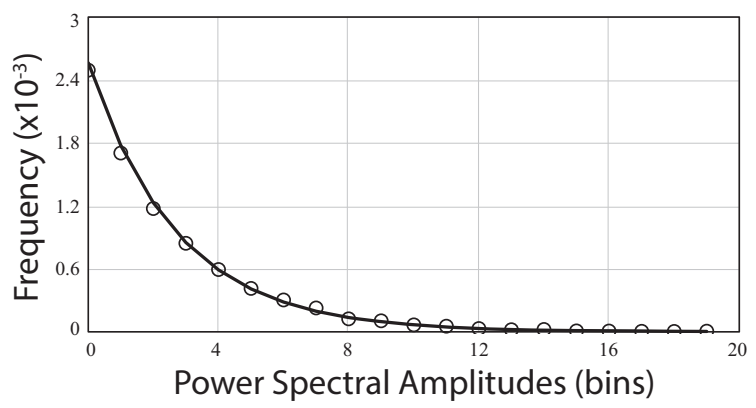

Fig. 9: Histogram of power spectral amplitudes $S_{j}^{(\mathrm{FFT})}$ (open circles) superposed by $E\left(\sigma_{d}^{2}\right)$ pdf (13) (solid line). The histogram comprises 9685 powers distributed over 20 bins of width $3.372 \times 10^{-7}$

where $\mu_{s} \equiv\langle S\rangle=\sigma_{d}^{2}[2]$. Figure 9 shows a histogram of $S_{j^{\prime}}^{(\mathrm{FFT})}$ superposed by the theoretical pdf (13) with slope parameter $\mu_{s}=(4.230 \pm 0.403) \times 10^{-7}$ (to be compared with $\left.\sigma_{d}^{2}=4.137 \times 10^{-7}\right)$ obtained by ML fit to the distribution $\left\{\ln S_{j}^{\mathrm{FFT}}\right\}$. The exponential fit is visually perfect and satisfies a chi-square test with $p\left(\chi_{18}^{2}\right)=0.147$.

To determine whether the four power spectra contained any statistically significant frequencies, a Walker-Fisher (WF) test was applied [2,13]. The WF test, based on order statistics [19], determines the probability that at least one element of the set $\left\{S_{j}\right\}$ exceeds the largest observed value $S_{\max }$

$$
P\left(S \geq S_{\max }\right)=1-\left(1-e^{-S_{\max } / \mu_{S}}\right)^{\frac{N}{2}} .
$$

The WF test led in the case of each power spectrum, except $S^{(\mathrm{FFT})}$, to a probability (14) greater than the conventional $5 \%$ threshold of statistical significance as shown in table 1 . However, the physical significance of any calculation of probability is the expectation to which it leads. Thus, given $\frac{1}{2} M=8192$ elements in the spectrum $S^{(\mathrm{FFT})}$, one expects that $(8192)\left(1.408 \times 10^{-4}\right)=1.15$ will fail the WF test purely by chance. A single failure, in fact, is what was observed. The violating peak occurred at harmonic $j=3$, corresponding to a period of 5461 points/cycle or
Table 1: WF tests of power spectrum of residuals.

\begin{tabular}{|l|l|l|}
\hline Power spectrum & WF probability & Series length \\
\hline$S^{(\mathrm{WK})}$ & 0.099 & $N=19370 \mathrm{pt}$ \\
\hline$S^{(\mathrm{BT})}$ & 0.452 & $N=19370 \mathrm{pt}$ \\
\hline$S^{(\mathrm{FT})}$ & 0.830 & $T=1001 \mathrm{~d}$ \\
\hline$S^{(\mathrm{FFT})}$ & $1.408 \times 10^{-4}$ & $M=16384 \mathrm{pt}$ \\
\hline
\end{tabular}

a temporal period of $205.4 \mathrm{~d}$, which has no apparent geophysical or astrophysical significance.

Conclusions and limits of sensitivity. - This paper reports a statistical analysis of the decay of ${ }^{54} \mathrm{Mn}$, one purpose of which is to search for evidence of a periodic forcing of the decay rate attributable to solar activity as claimed in recent publications. The analysis, covering a time span of about 3.6 half-lives, examined the residuals of the logarithms of ${ }^{54} \mathrm{Mn}$ activity measured by current in an ionisation chamber. The residuals were tested for their variation in time, autocorrelation, power spectrum, and agreement with theoretical distribution functions. The outcome of each test was in excellent accord with expectations of standard nuclear physics.

In particular, no evidence was found of periodicity in the ${ }^{54} \mathrm{Mn}$ decay rate nor of solar-correlated spikes in the decay curve. The IRMM data did not cover the $\sim 3 \mathrm{y}$ measurement period uniformly and may have missed a fluctuation of a kind reported in [8]. That it would have missed all such fluctuations during $3 \mathrm{y}$ is deemed unlikely. Moreover, the Sun's irradiance from more than 10 million normal modes is known to affect nearly all engineering systems [20]; reported correlations with solar eruptions, therefore, more likely signify environmental variations of detector efficiency [21] than a direct influence on nuclear decay.

To determine the sensitivity of this analysis to reveal external periodic forcing, the tests were performed on modified data sets whereby each activity measurement $x_{n}$ observed at $t_{n}$ in the original time series was multiplied by a factor of the form $\exp \left(-\alpha \lambda t_{n} \cos \left(2 \pi t_{n} / T_{1}\right)\right)$. Since the observed ${ }^{54} \mathrm{Mn}$ activity was shown to follow nearly perfectly an exponential decay law (see fig. 1), the modifications to the data simulated the activity of a ${ }^{54} \mathrm{Mn}$ sample whose decay rate took the time-dependent form

$$
\Lambda_{n}=\lambda\left(1+\alpha \cos \left(2 \pi t_{n} / T_{1}\right)\right)
$$

with anomalous relative amplitude $\alpha$ and period $T_{1}$, as discussed in $[1,12]$.

Figure 10 shows a plot of residuals as a function of (A) time and (B) point number for parameters $\alpha=5 \times 10^{-4}$ and $T_{1}=360 \mathrm{~d}$. A period close to $1 \mathrm{y}$ was chosen to address reports of anomalies correlated with the Earth's orbit. Figure 11 shows the corresponding autocorrelation up to maximum lag $K=18000$, calculated by eq. (7) with 

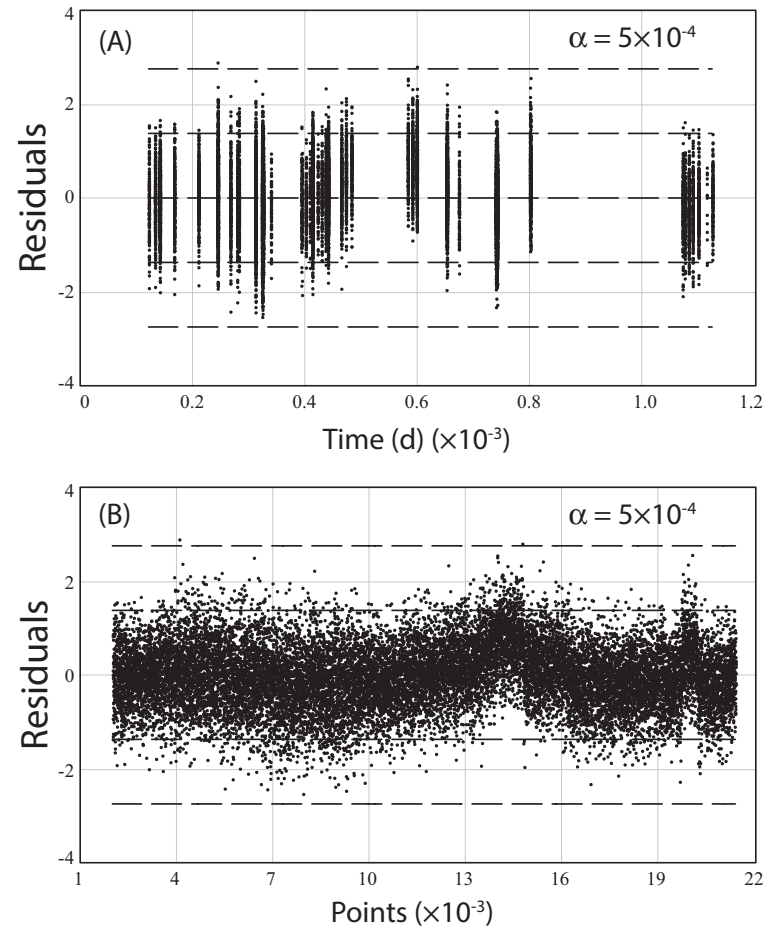

Fig. 10: Simulated ${ }^{54} \mathrm{Mn}$ residuals (in units of $\sigma_{r}$ ) with decay rate (15) as a function of (A) time (d) and (B) point number. $N=19370$ points, amplitude $\alpha=5 \times 10^{-4}$, period $T_{1}=360 \mathrm{~d}$.

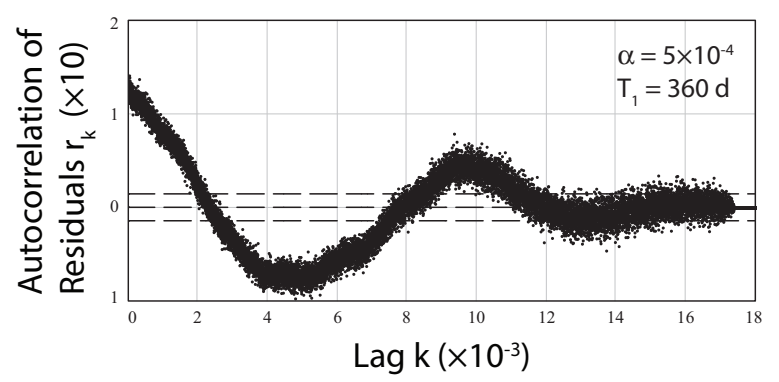

Fig. 11: Simulated ${ }^{54} \mathrm{Mn}$ autocovariance with decay rate (15) as a function of lag (in points). $\quad N=19370$, amplitude $\alpha=5 \times 10^{-4}, T_{1}=360 \mathrm{~d}$.

prefactor $(N-k)^{-1}$. Not only are the anomalous patterns visually striking, but the four power spectra strongly fail the WF tests $\left(P\left(S \geq S_{\max }\right) \approx 0\right)$, revealing a statistically significant peak at harmonics corresponding to 360 d. Although less obvious, anomalies in the modified data sets were still detectable statistically at an amplitude of $\alpha=1 \times 10^{-4}$, which is at least an order of magnitude smaller than deviations from exponential decay claimed in the literature.

$$
* * *
$$

The author thanks Dr Stefan Pommé of the Institute for Reference Materials and Measurements for providing the data of ${ }^{54} \mathrm{Mn}$ activities.

\section{REFERENCES}

[1] Silverman M. P., J. Mod. Phys., 6 (2015) 1533.

[2] Silverman M. P. and Strange W., EPL, 87 (2009) 32001.

[3] Cooper P. S., Astropart. Phys., 31 (2009) 267.

[4] http://www.nucleide.org/DDEP_WG/Nuclides/Mn-54_ tables.pdf.

[5] Jenkins J. H. and Fischbach E., Astropart. Phys., 31 (2009) 407.

[6] Cartwright J., http://physicsworld.com/cws/ article/news/2008/oct/02/the-mystery-of-thevarying-nuclear-decay.

[7] Castelvecchi D., Sci. News, 174 (2008) 21.

[8] Mohsinally T. et al., Astropart. Phys., 75 (2016) 29.

[9] Magill J. and Galy J., Radioactivity, Radionuclides, Radiation (Springer) 2005, Chapt. 4.

[10] Bambynek W. et al., Rev. Mod. Phys., 49 (1977) 77.

[11] van Ammel R., Paepen J., Pomme S. and Sibbens G., Appl. Radiat. Isot., 68 (2010) 2387.

[12] Silverman M. P., EPL, 110 (2015) 52001.

[13] Silverman M. P., A Certain Uncertainty: Nature's Random Ways (Cambridge University Press) 2014.

[14] Kendall M., Stuart A. and Ord J. K., The Advanced Theory of Statistics, Vol. 3, 4th edition (Macmillan) 1983, pp. $443-445$.

[15] Ghil M. et al., Rev. Geophys., 40 (2002) 1.

[16] Bendat J. S. and Piersol A. G., Measurement and Analysis of Random Data (Wiley) 1966.

[17] Chatfield C., The Analysis of Time Series, 6th edition (Chapman and Hall) 2003, p. 131.

[18] Cooley J. W. and Tukey J. W., Math. Comput., (1965) 297.

[19] Mood A. M., Graybill F. A. and Boes D. C., Introduction to the Theory of Statistics, 3rd edition (McGraw-Hill), pp. 251-265.

[20] Thomson D. J. et al., Proc. IEEE, 95 (2007) 1085.

[21] Meier M. and Wieler R., Astropart. Phys., 55 (2015) 63. 